\title{
A Study on Giant Magnetoimpedance Effect in VITROVAC $6025 Z$ Micro-patterned Ribbons
}

\author{
Zhen Yang ${ }^{1, a}$, Tianqi Fan ${ }^{2}$ and Chong Lei ${ }^{2}$ \\ ${ }^{1}$ School of Physics and Electronic Engineering, Xinyang Normal University, Xinyang 464000, People's Republic of \\ China \\ ${ }^{2}$ Key Laboratory for Thin Film and Microfabrication of the Ministry of Education, Department of Micro/Nano \\ Electronics, School of electronic information and electrical engineering, Shanghai Jiao Tong University, Dong \\ Chuan Road 800, Shanghai 200240, People's Republic of China
}

\begin{abstract}
In this paper, micro-patterned cobalt-based amorphous ribbons with different structures are fabricated by MEMS technology, including bonding, lithography and electroplating. The commercial amorphous ribbons (VITROVAC 6025Z) are purchased from VACUUMSHMELZE $^{\circledR}$ company. The structures of micro-patterned ribbons are designed for meander shape for different turns (one turn, two turns and three turns). The effect of external magnetic field orientation and turns on GMI ratios were studied in the frequency range of 1-40 MHz. It is found that GMI ratios almost all present negative value, which is related to the material property, namely a large coercive force. Compared with the GMI ratios obtained at transverse external magnetic field, the maximum negative GMI ratio obtained at longitudinal external magnetic field is higher. The value of $-69.93 \%$ is obtained at a current frequency of 20 $\mathrm{MHz}$ and the magnetic field of $150 \mathrm{Oe}$. With the increase of the number of turns, the maximum positive GMI ratio increases from $0.5 \%$ for the one turn sample to the $2.8 \%$ for the sample with three turns. The maximum nagative GMI ratio is obtained by three-turns sample. The GMI character of VITROVAC $6025 \mathrm{Z}$ micro-patterned ribbon is significant and the microsensor is expected to be used in biological sensing field.
\end{abstract}

\section{Introduction}

Giant magnetoimpedance (GMI) effect is defined as a large change in the ac complex impedance of a soft ferromagnetic conductor upon the application of a dc magnetic field. It has drawn an intensively research activity by reason of promising technological applications based on the GMI phenomenon since its discovery $[1,2]$. Among various kinds of existing GMI materials, soft ferromagnetic amorphous ribbons are considered attractive candidates for making all kinds of GMI-based magnetic sensors [3-5], because they possess a stripe-like domain structure along the transverse direction, which is favourable for achieving GMI effect with a high degree of field sensitivity.

In recent years, the GMI effect of micro-patterned ribbon with tortuous structure is extensively investigated. Because meander-shaped micro-patterned ribbon possesses high GMI ratio compared that of the ribbon with single strip structure [6-7]. Cobalt-based amorphous alloys, e.g., Vitrovac,

\footnotetext{
${ }^{\text {a } C o r r e s p o n d i n g ~ a u t h o r ~: ~ z h c 025 @ s j t u . e d u . c n ~}$
} 
exhibit a high GMI level, a high sensitivity to an applied magnetic field [8], which makes them promising GMI materials.

So, in this context, we employ micro electro-mechanical system (MEMS) technology to fabricate micro-patterned cobalt-based amorphous ribbons (VITROVAC 6025Z) with tortuous shape for different turns. The GMI ratio of the ribbons are investigated at different magnetic fields (1-150 Oe) and frequencies (1-40 MHz). The influence of external magnetic field orientation and turns on GMI ratios are observed. The results show that the GMI ratios obtained at longitudinal external magnetic field is higher than that obtained at transverse external magnetic field. And with the increase of the number of turns, the maximum of the GMI ratio increases from $31.2 \%$ for the one turn sample to the $69.93 \%$ for the sample with three turns.

\section{Experimental procedures}

\subsection{Soft magnetic materials}

In this section, the micro-patterned GMI sensor elements are made of a cobalt-based commercials amorphous ribbon (VITROVAC 6025Z). The thickness of ribbon is $25 \mu \mathrm{m}$. The main physical and magnetic properties of the ribbons are listed in Table 1.

Table 1. The main physical and magnetic properties of commercials ribbon.

\begin{tabular}{|c|c|}
\hline Parameters & $\mathbf{6 0 2 5 Z}$ \\
\hline Saturation induction $\mathrm{B}_{\mathrm{S}}(\mathrm{T})$ & 0.55 \\
\hline Saturation magnetostriction constant & $-3 \times 10^{-7}$ \\
\hline Maximum permeability $\mu_{\max }$ & $1.03 \times 10^{5}$ \\
\hline Coercivity $\mathrm{H}_{\mathrm{c}}(\mathrm{A} / \mathrm{m})$ & 2.84 \\
\hline
\end{tabular}

\subsection{Fabrication of micro-patterned ribbons}

The micro-patterned ribbons with tortuous shape (one-turn, two-turn, and three structures) (as shown in Figure. 1) were designed with width of $500 \mu \mathrm{m}$ and length of $10 \mathrm{~mm}$. The intervals of tortuous shape were $60 \mu \mathrm{m}$. Each 'n' shape in the meander structures was called one turn. Before MEMS processing, the ribbons were mechanical polished. After mechanical polishing, the thickness of the ribbon was measured by surface profiler, and was $15 \mu \mathrm{m}$.

The ribbons with different structures were fabricated by bonding, photolithography, chemical etching and electroplating methods. The manufacturing process consisted of the following steps:

I) Cleaning substrate: At first, the glass substrate were cleaned by ultrasonic in acetone solution and alcohol in turn for $10 \mathrm{~min}$, and then the substrates were rinsed with deionized water and alcohol, at last it was dried using a stream of nitrogen gas;

$\Pi$ П) Bonding ribbon: the ribbon was bound on clean glass substrates using an epoxy adhesive, during binding we should try to ensure tight bonding and no air between the ribbon and the substrate;

Ш) Lithography: photoresist was spun on ribbon with the thickness of $15 \mu \mathrm{m}$, and then the ribbon was patterned with a mask by UV lithography;

IV) Chemical etching: the ribbon was etched in acidic mixed solution $\left(\mathrm{HNO}_{3}: \mathrm{HCl}: \mathrm{H}_{2} \mathrm{O}_{2}: \mathrm{H}_{2} \mathrm{O}=\right.$ 1:2:4:8) for $5 \mathrm{~min}$. The error of $\pm 2.5 \mu \mathrm{m}$ was found after etching process and it was considered to be acceptable. At last, the photoresist was removed;

V) Electroplating $\mathrm{Cu}$ electrode: Before electroplating, firstly, polyimide was filled the space between neighbor segments in order to avoid the adverse effects of the nonuniform surface, and then 
$\mathrm{Cr} / \mathrm{Cu}$ seed layer was deposited on patterned ribbon by $\mathrm{RF}$ sputtering, at last $\mathrm{Cu}$ electrodes with thickness of $20 \mu \mathrm{m}$ were electroplated;

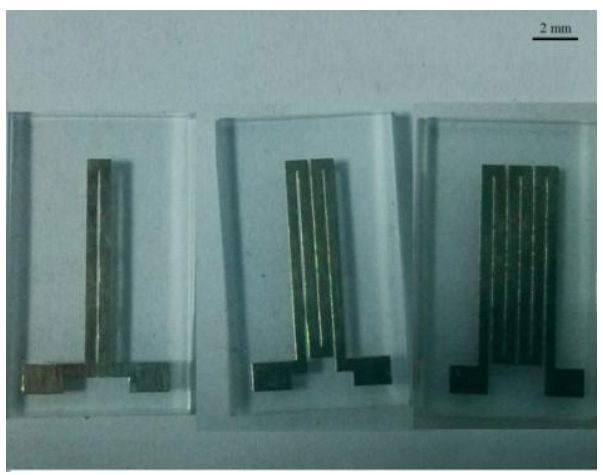

Figure 1. The top view of fabricated micro-patterned Co-based amorphous ribbon.

\subsection{Measuring methods}

The GMI effects in micro-patterned ribbon were measured by an impedance analyzer (HP4194A) in the frequency range of 1-40 MHz with the constant current amplitude of 10mA. An external magnetic field $\left(\mathrm{H}_{\mathrm{ex}}\right)$ of 1-150 Oe was applied along transverse and the longitudinal direction of the sample. Magnetic field is got from a permanent, the magnitude of that was controlled through changing the distance between the permanent and micro-patterned ribbon. The permanent slowly closed to the ribbon along the central axis of ribbon. Magnetic fields at different locations were measured strictly by a magnetometer, so the different marked locations standed for different magnetic fields [6].

Figure. 2 shows schematic diagram of (a) transverse and (b) longitudinal magnetic field. The GMI ratio is defined as: GMI ratio $(100 \%)=100 \% \times\left[\mathrm{Z}(\mathrm{H})-\mathrm{Z}\left(\mathrm{H}_{0}\right)\right] / \mathrm{Z}\left(\mathrm{H}_{0}\right)$, where $\mathrm{Z}(\mathrm{H})$ and $\mathrm{Z}\left(\mathrm{H}_{0}\right)$ are the magnetoimpedance with and without magnetic field respectively.

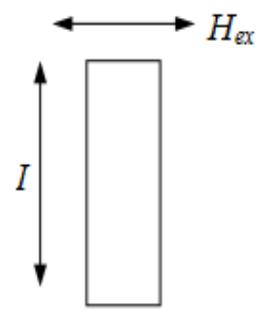

(a)

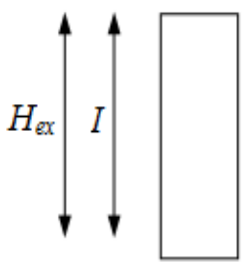

(b)

Figure 2. Schematic diagram of (a) transverse and (b) longitudinal magnetic field.

\section{Results and discussion}

\subsection{Effect of external magnetic field on GMI}

Figure. 3 shows field dependence of the GMI ratio of the tortuous-shaped ribbons at different frequency with the transverse fields. In sample testing, an external magnetic field of 1-150 Oe is applied along the transverse direction of the meander ribbon and is parallel to the transverse anisotropy direction of the sample. 
The positive GMI ratio is very small and appear at small external magnetic field (1-5 Oe). When the external magnetic field is greater than 5 Oe, the GMI ratio all presented negative value. The maximum negative GMI ratio is obtained at frequency $\mathrm{f}=20 \mathrm{MHz}$. On the whole, with increasing the applied magnetic field $\mathrm{H}_{\mathrm{ex}}$, the magnitude of the GMI ratio first reaches a slight sharp peak and then gradually decreases to zero and even negative values. The negative values become more and more big with increasing $\mathrm{H}_{\mathrm{ex}}$. In the measurement range, the maximum negative GMI ratio is $-41.6 \%$ obtained at $\mathrm{H}_{\mathrm{ex}}=150 \mathrm{Oe}$ and frequency $\mathrm{f}=20 \mathrm{MHz}$. Our preliminary forecast a maximum negative GMI peak will appear quickly.

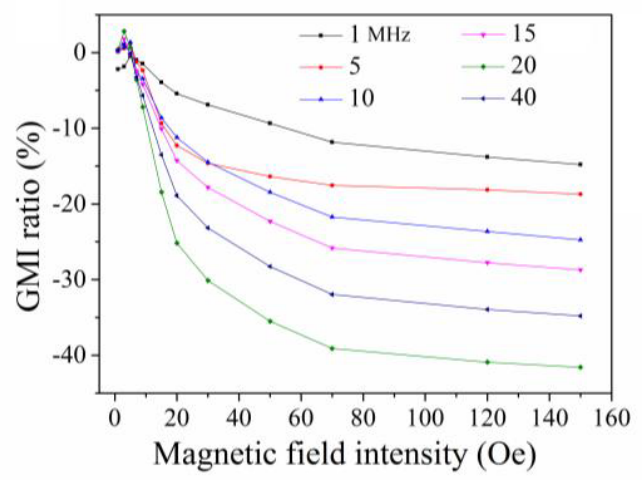

Figure 3. Field dependence of the GMI ratio of the tortuous-shaped ribbons at different frequency with the transverse fields.

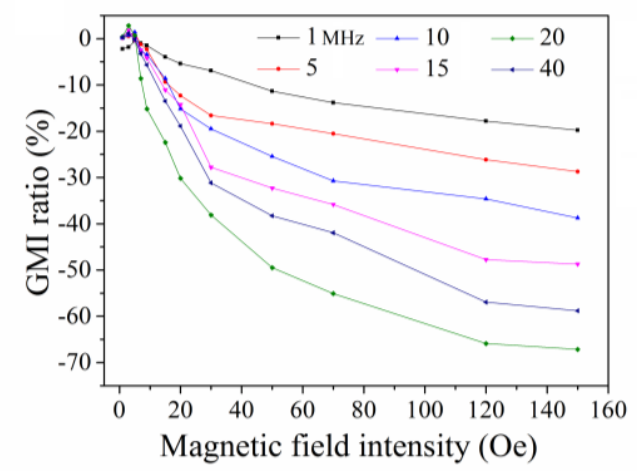

Figure 4. Field dependence of the GMI ratio of the tortuous-shaped ribbons at different frequency with the longitudinal fields.

Figure. 4 shows field dependence of the GMI ratio of the tortuous-shaped ribbons at different frequency with the longitudinal fields. Compared with figure.3, in the measurement range, the maximum negative GMI ratio is $-69.93 \%$ obtained at $\mathrm{H}_{\mathrm{ex}}=150 \mathrm{Oe}$ and frequency $\mathrm{f}=20 \mathrm{MHz}$. The phenomenon of GMI change has been observed by Sommer [9]. Sommer indicates that a longitudinal GMI spectrum of soft magnetic materials with high transverse permeability has the typical characteristic. The GMI curves in Figure. 4 agree well with this typical characteristic, which can be explained by the magnetization rotation model and it goes as follows: the rotational magnetic permeability related to the GMI ratio increases with the increase in Hex and reaches a maximum value as $\mathrm{H}_{\mathrm{ex}}=\mathrm{H}_{\mathrm{K}}$, where $\mathrm{H}_{\mathrm{ex}}$ is perpendicular to the transverse anisotropy direction of the sample and $\mathrm{H}_{\mathrm{K}}$ is the anisotropy field. Thereafter, the permeability decreases with $\mathrm{H}_{\mathrm{ex}}$ and the GMI ratio gradually drops. The difference between the longitudinal and the transverse GMI effect is due to the two main reasons: one is the easy axis deviates from the transverse direction of the sample; the other is the 
demagnetizing factor $\mathrm{D}_{\perp}$ effect on the longitudinal permeability. Compared with the previous research [6], we design more appropriate size. The error of $\pm 2 \mu \mathrm{m}$ was found and smaller than previous error $( \pm 5.5 \mu \mathrm{m})$ after etching process, and it was considered to be acceptable. The small error leads to high GMI performance.

\subsection{Effect of turn on GMI}

Figure. 5 shows field and frequency dependence of the longitudinal GMI ratio of the tortuous-shaped ribbons with different turns. And the maximum positive GMI ratios obtained at a field of 3 Oe increases from $0.5 \%$ for the one turn sample to $2.8 \%$ for the sample with three turns. The maximum negative GMI ratios were obtained at different frequencies for samples with different turns. The maximum negative GMI ratios of one-turn, two-turns and three-turns samples were $-34.8 \%,-50.9 \%$ and $-66.9 \%$, obtained at $4.5 \mathrm{MHz}, 10.5 \mathrm{MHz}$ and $20 \mathrm{MHz}$ respectively.

The results show that the ribbons with tortuous shape of three turns acquire the maximum positive GMI and the maximum negative GMI ratios at a relatively small magnetic field and a higher frequency. This is related to the material property, namely a large coercive force. Besides, three-turns samples has more complex mutual inductance. When the ac current flows through the tortuous ribbon, just as for the flux in each longitudinal and transverse straight line in a plane inductance, the fluxes will correlate with each other by mutual inductance of the magnetic chains. The more turns of the meander structure, the more intense the interaction becomes. As a result, these effects lead to the increase in $\mathrm{L}_{\mathrm{m}}$ and $\mathrm{Z}$. More turns mean to stronger mutual inductance and higher GMI ratio, but big size is not suitable for miniaturization of microsensor.
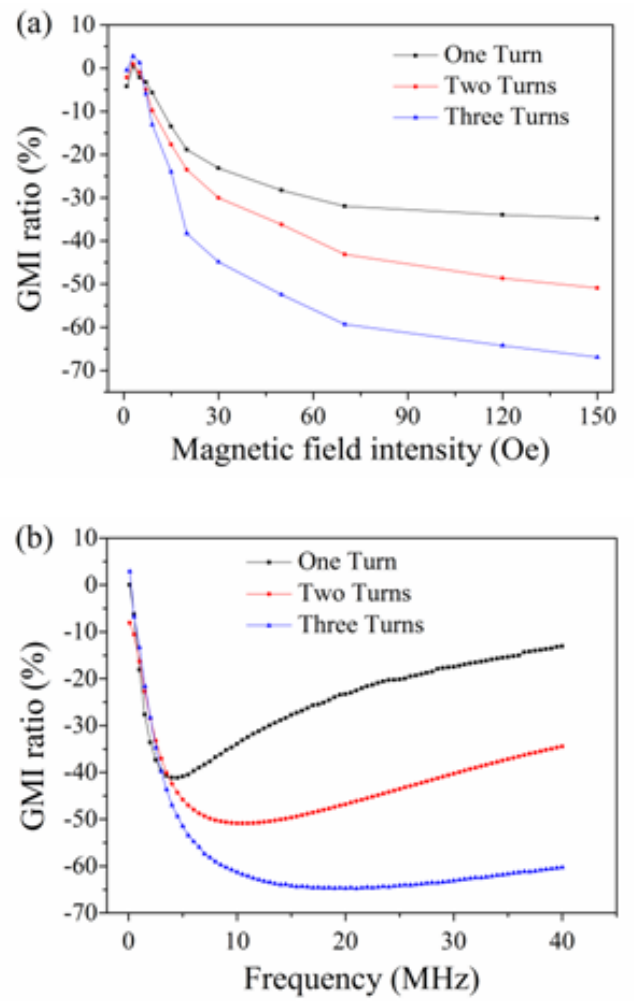

Figure 5. (a) Field and (b) frequency dependence of the GMI ratio of the tortuous-shaped ribbons with different turns. 


\section{Conclusion}

In conclusion, the GMI ratios of VITROVAC $6025 \mathrm{Z}$ micro-patterned ribbons with meander structure for different turns almost all present negative value, which is related to the material property, namely a large coercive force. The small positive GMI ratio only appear when the external magnetic field is in the range of 1-5 Oe. The effect of external magnetic field orientation and turns on GMI ratios were studied in the frequency range of $1-40 \mathrm{MHz}$. It is found that the maximum negative GMI ratios obtained at longitudinal external magnetic field is higher than that obtained at transverse external magnetic field. The value of $-69.93 \%$ was obtained at a current frequency of $20 \mathrm{MHz}$ and the magnetic field of 150 Oe. With the increase of the number of turns, the maximum positive GMI ratio increases from $0.5 \%$ for the one turn sample to the $2.8 \%$ for the sample with three turns. The maximum nagative GMI ratio was obtained by three-turns sample. The GMI character of VITROVAC $6025 \mathrm{Z}$ micro-patterned ribbons is significant and the microsensor is expected to be used in biological sensing field.

\section{Acknowledgments}

This work was supported by The National Natural Science Foundation of China (No. 61273065),

\section{References}

1. L. Kraus, M. Vázquez, M.Knobel, K. H. J. Buschow, Nort-Holland (2003).

2. V. Zhukova, A. Chizhik, A. Zhukov, A. Torccunov, V. Larin, González, IEEE Trans. Magn. 38, 3090 (2002)

3. A. Chaturvedi, K. Stojak, N. Laurita, P. Mukherjee, H. Srikanth, and M. -H. Phan, J. Appl. Phys. 111, 07 (2012).

4. Y. Zhang, J. Dong, E. X. Feng, C. Q. Luo, Q. F. Liu, J. B. Wang, Chin. Phys. Lett. 30, 037501 (2013).

5. L. González, J. Bonastre, T. Sánchez, J. D. Santos, M. L. Sánchez, A. Chizhik, L. Domínguez, M. Ipatov, V. Zhukova, A. Zhukov, J. González, J. J. Suñol, B. Hernando, IEEE Trans. Magn. 48, 4375 (2012).

6. Z. Yang, J. Lei, C. Lei, Y. Zhou, T. Wang, Appl. Phys. A. 1-5 (2014)

7. L. Chen, Y. Zhou, C. Lei, Z. M. Zhou, W. Ding, Journal of Physics D: Applied Physics. 42(14), 145005 (2009)

8. G. V. Kurlyandskaya, M. L. Sanchez, B. Hernando, et al., Appl. Phys. Lett. 82, 3053 (2003).

9. R. L. Sommer, C. L. Chine, J Appl Phys. 79, 5139 (1996). 